\title{
Faculty Learning Communities e Comunità di Pratica per lo sviluppo professionale del docente. L'esperienza dell'Università di Genova
}

\section{Faculty Learning Communities and Communities of Practice for Faculty Development. The University of Genoa's experience}

\author{
Antonella Lotti*
}

\begin{abstract}
Riassunto
Questo articolo offre l'occasione di riflettere su cosa siano le Faculty Learning Communities (FLC) e le Comunità di Pratica (CdP) all'interno dei contesti universitari, con particolare attenzione ai gruppi di docenti che si incontrano con regolarità per riflettere sulle proprie pratiche di insegnamento.

Lo scopo di questo saggio è di definire cosa siano questi due tipi di gruppi di confronto e lavoro, illustrarne le origini e le caratteristiche, descrivere qualche esempio di FLC e CdP, tratteggiare le teorie di riferimento e riportare l'esperienza dell'Università di Genova, che realizza alcune Comunità di Pratica dal 2017, alla presenza dei risultati di un questionario somministrato a cinquanta docenti genovesi. Per concludere, e con riferimento a un articolo recente dedicato alle CdP per lo sviluppo delle competenze didattiche dei docenti di area medica e sanitaria, verranno riportate le dodici raccomandazioni utili per avviare e mantenere sempre attive ed efficaci le CdP nei contesti universitari.
\end{abstract}

Parole Chiave: Faculty Learning Communities; Comunità di Pratica; Faculty Development.

\begin{abstract}
This article offers an opportunity to reflect on what Faculty Learning Communities (FLC) and Communities of Practice (CoP) are within university contexts, with particular attention to groups of scholars who meet regularly to
\end{abstract}

\footnotetext{
*Università di Modena e Reggio Emilia. E-mail: antonella.lotti@unimore.it.
} 
reflect on their teaching practices. The purpose of this paper is to define what Faculty Learning Communities and Community of Practice are, to illustrate their origins and characteristics, to describe some examples of FLC and CoP, to outline their theoretical framework, to report the University of Genoa's experience about some Communities of Practice launched in 2017. Finally, some tips for implementing a community of practice for faculty development will be described.

Keywords: Faculty Learning Communities; Community of Practice; Faculty Development

\section{Introduzione}

In questi ultimi anni si è registrato un interesse crescente verso le strategie utilizzate per sviluppare le competenze didattiche dei docenti universitari. Accanto ai metodi più diffusi e tradizionali, quali i seminari e i workshop, proposti da un organismo centrale dell'ateneo preposto al Faculty Development, si stanno diffondendo esperienze che partono dal basso e che sono più informali: le Faculty Learning Communities (FLC) e le Comunità di Pratica (CdP).

\section{Faculty Learning Communities}

Faculty Learning Communities (FLCS) letteralmente significa Comunità di apprendimento di docenti universitari, o membri di Facoltà, ed è stato coniato dall'Università di Miami negli Stati Uniti, alla fine degli anni Settanta, per definire quei gruppi di docenti che si creavano ogni anno con l'obiettivo di affrontare argomenti dedicati all'insegnamento e all'apprendimento (Cox, 2004).

Una Faculty Learning Community è un gruppo interdisciplinare di docenti universitari che si incontrano su base volontaria e si impegnano a pianificare e realizzare un programma formativo annuale dedicato al miglioramento dell'insegnamento e apprendimento. Ogni gruppo, di solito, è composto da un massimo di quindici partecipanti, è guidato da un facilitatore, può organizzare seminari, ritiri residenziali, o altre attività che favoriscano l'apprendimento, inteso come approfondimento teorico e scientifico della didattica universitaria e la costruzione di una piccola comunità. 
Nell'ambito delle attività di Faculty Development, ogni anno vengono creati due tipi di Faculty Learning Communities: quelle dedicate a un tema o a una coorte.

Le FLCs organizzate per coorte sono quelle composte da un gruppo omogeneo di partecipanti: docenti giovani, docenti a metà carriera e fine carriera, direttori dei dipartimenti, presidi di scuole o facoltà. Questi gruppi si occupano di insegnamento e apprendimento, e cercano di far superare il sentimento di solitudine e isolamento che spesso tocca alcuni docenti universitari nell'espletamento dei propri compiti didattici.

Le FLCs dedicate a un tema, invece, pianificano un programma formativo mirato a rispondere a un bisogno formativo o a un'opportunità offerta a un determinato corso di studio o dipartimento. Di solito sono i docenti stessi, o i tecnici amministrativi, che propongono un elenco di argomenti al direttore del programma FLCs, il quale, a sua volta, pubblica un bando aperto a tutti i docenti dell'ateneo, chiedendo agli interessati di iscriversi. Un argomento può durare un anno, o più anni, e si conclude quando il gruppo ha raggiunto i suoi obiettivi formativi.

I temi affrontati in queste FLCs possono essere, ad esempio, la trasformazione dei corsi con approccio multiculturale, la tecnologia collaborativa, l'apprendimento e l'insegnamento online, il Problem Based Learning, insegnare e apprendere l'etica nei laboratori, la psicologia dell'apprendimento, insegnare in una lingua straniera, insegnare e apprendere in classi molto numerose, valutazione formativa e certificativa.

Le FLCs, di solito, durano per un periodo che va oltre i sei mesi, sono su base volontaria, si incontrano in un ambiente che favorisce l'apprendimento, trattano progetti individuali o di gruppo, adottano il ciclo di Kolb dedicato all'apprendimento esperienziale (Kolb, 1984), sviluppano l'empatia tra i partecipanti, prendono le decisioni tramite il consenso, instaurano un clima di fiducia e un atteggiamento non giudicante, affrontano anche problemi complessi, cercano di dare energia ai partecipanti e renderli più competenti.

Le FLCs hanno ormai raggiunto una esperienza quarantennale, sono state studiate e valutate da alcuni studiosi, i quali, oltre ad evidenziarne le ricadute sui docenti partecipanti e sui loro studenti, sono riusciti anche a identificare quali sono i requisiti che rendono una FLC efficace (Cox, 2004).

Tra le ricadute, Milton J. Cox cita:

- riduzione dei problemi stress-correlati nei giovani docenti con contratti a tempo determinato, che evidenziavano isolamento e mancanza di comunità di riferimento; 
- maggiore disponibilità verso altri approcci didattici proposti;

- riconoscimenti pubblici con premi in ambito didattico;

- aumento di rispetto e sensibilità verso il punto di vista altrui, sia degli studenti che dei docenti;

- aumento negli studenti di alcune competenze trasversali (problem solving, collaborazione, lavoro in gruppo) e sviluppo di alcuni atteggiamenti, tra cui, maggior entusiasmo verso alcuni argomenti, correlato alla passione dei propri docenti;

- pianificazione di nuovi curricula.

Con riferimento all'ultimo punto, si è visto che le FLCs favoriscono l'introduzione di innovazioni didattiche e cambiamenti importanti a livello curriculare nei corsi di studio (Engin \& Atkinson, 2015; Yonge \& Davidson, 2017).

\section{Le Comunità di Pratica}

Le FLCs fanno parte di una strategia formativa, utilizzata nelle organizzazioni, che utilizza un particolare tipo di gruppo come luogo di scambio e apprendimento reciproco: la Comunità di Pratica $(\mathrm{CdP})$ teorizzata da Etienne Wenger nel 1998 (Wenger, 1998). Anche Milton Cox scriveva che le FLCs sono un particolare genere di comunità di pratica (Cox, 2004).

La Comunità di Pratica $(\mathrm{CdP})$ è una rete sociale di persone che condividono credenze, valori, storie ed esperienze, focalizzate su una pratica comune e/o su un'impresa condivisa. Per poter parlare di CdP, Etienne Wenger scrive che ci vogliono tre elementi: un campo tematico, una comunità e una pratica, infatti una CdP si riferisce a un gruppo di persone che condividono un campo di interesse. Questi individui sono impegnati in un dato campo, valorizzano la loro esperienza collettiva e apprendono gli uni dagli altri.

Le CdP sono gruppi di persone che condividono una preoccupazione, una serie di problemi o una passione per un argomento e che approfondiscono le loro conoscenze e competenze in questo settore interagendo su base continuativa. Queste persone non necessariamente lavorano insieme ogni giorno, ma si incontrano perché trovano valore nelle loro interazioni. Mentre trascorrono del tempo insieme, in genere condividono informazioni e consigli. Si aiutano a vicenda a risolvere i problemi. Discutono delle loro situazioni, delle loro aspirazioni e dei loro bisogni. Riflettono su problemi comuni, esplorano idee e fungono da cassa di risonanza. Nel tempo, sviluppano una prospettiva unica sul loro argomento, nonché un corpus di conoscenze, pratiche e approcci comuni. Sviluppano anche relazioni personali e 
modi stabiliti di interagire. Possono persino sviluppare un senso comune di identità. Diventano una comunità di pratica (Wenger, 1998).

Le Comunità di Pratica sorgono alla fine degli anni Ottanta nel mondo dell'industria delle automobili, presso la Chrysler Corporation, la quale, dovendo sveltire i processi ideativi e realizzativi per poter proporre un nuovo veicolo sul mercato, e competere con maggiore efficienza con le altre case automobilistiche, decise di cambiare il modello organizzativo per unità funzionali, che prevedevano unità di progettazione, ingegnerizzazione, produzione e vendita, tutte operanti a compartimenti stagni in modo superspecializzato.

Si decise di creare "piattaforme" organizzative specificatamente dedicate alle automobili. Queste "piattaforme" si configuravano come strutture orientate al prodotto, trasversali alle funzioni: ad esempio automobili di grandi dimensioni, utilitarie, furgoni, camion e Jeep. Ogni "piattaforma" era responsabile di tutti le fasi di sviluppo associate all'intero veicolo (Wenger, McDermott \& Snyder, 2002).

In questo modo i tempi si ridussero per produrre un veicolo, ma sorse il problema che negli stessi gruppi di lavoro si proponevano spesso problemi simili e i vari professionisti, per risolverli, si rivolgevano spontaneamente ai vecchi colleghi con cui avevano lavorato nelle medesime aree funzionali. I manager decisero di sostenere e incoraggiare questi incontri informali, che si rivelavano molto efficaci e produttivi, e decisero di chiamarli TechClub. Questi TechClub sono l'origine delle Comunità di Pratica. Gli ingegneri che partecipavano a questi gruppi di scambio realizzarono che il tempo passato insieme ai colleghi era ben investito, perché essi risolvevano i problemi in modo più rapido, avevano maggior fiducia nei nuovi progetti, venivano a conoscenza in anteprima di cosa stavano scoprendo i colleghi ed erano maggiormente motivati a esplorare nuove tecnologie. Questo modello di TechClub ha dato origine a centinaia di altri TechClub in Crysler, ed è stato poi diffuso anche in altre grandi multinazionali come la Shell Oil, la McKinsey \& Company e così via. La Comunità di Pratica è quindi costituita da un gruppo di persone che condividono lo stesso campo di interesse, che hanno una conoscenza tacita, basata sulla pratica, e che la condividono rendendola un apprendimento sociale.

Le CdP si possono sviluppare autonomamente, ma per crescere bene e in salute hanno bisogno di impegno, di coinvolgimento volontario e di una leadership interna. Wenger e collaboratori (Wenger et al., 2002) dicono che per far crescere una Comunità di Pratica bisogna coltivarla, e usano proprio la metafora 
della coltivazione di una pianta. Essi affermano, sulla base della loro esperienza, che le Comunità di Pratica che funzionano bene dovrebbero seguire i seguenti sette principi:

- progettare l'evoluzione;

- aprire il dialogo tra prospettive interne ed esterne;

- favorire differenti livelli di partecipazione;

- sviluppare spazi di comunità sia pubblici che privati;

- concentrarsi sul valore;

- combinare esperienze familiari ed eventi inconsueti;

- dare ritmo alla continuità.

Come ogni gruppo sociale, anche la Comunità di Pratica nasce, si sviluppa e si conclude, vivendo varie fasi evolutive, che in questo contesto vanno pianificate e accompagnate da un facilitatore o coordinatore della comunità, il cui ruolo è ritenuto cruciale. Il coordinatore della comunità di solito è un partecipante che aiuta la comunità a mettere a fuoco il suo campo tematico, a mantenere vive le relazioni e a sviluppare la sua pratica. Svolge alcune funzionichiave quali la pianificazione e la facilitazione della comunità e lo sviluppo dei suoi membri; identifica gli eventuali problemi rilevanti per il campo tematico; aiuta a far crescere la pratica utilizzando le conoscenze di base e quelle emerse dal campo, le buone pratiche e tutte le occasioni di apprendimento; valuta lo stato di salute della comunità e il suo contributo ai membri dell'organizzazione; gestisce i rapporti tra la comunità e l'organizzazione nella quale è posta.

Le Comunità di Pratica rappresentano oggi una forma di apprendimento sul posto di lavoro molto diffusa, che può assumere forme, nomi e stili diversi, ma che comunque rappresenta una importante esperienza di gestione della conoscenza.

\section{Le Comunità di Pratica per il Faculty Development}

Nella letteratura dedicata al Faculty Development vi sono molti riferimenti alle Comunità di Pratica. Yvonne Steinert colloca la Comunità di Pratica tra gli approcci formativi informali che si svolgono in gruppo (Figura 1) e riporta alcuni studi dedicati alle CdP per la formazione dei docenti di area medica (Steinert, 2014). Alcune studiose olandesi affermano che vi è uno spostamento graduale, nel Faculty Development, dalla formazione formale, svolta in workshop, verso la formazione informale nei luoghi di lavoro, laddove la didattica viene 
svolta, e che le comunità di pratica di docenti sono molto efficaci (Schreurs et al., 2016).

Fig. 1 - Approcci del Faculty Development (Steinert, 2010)

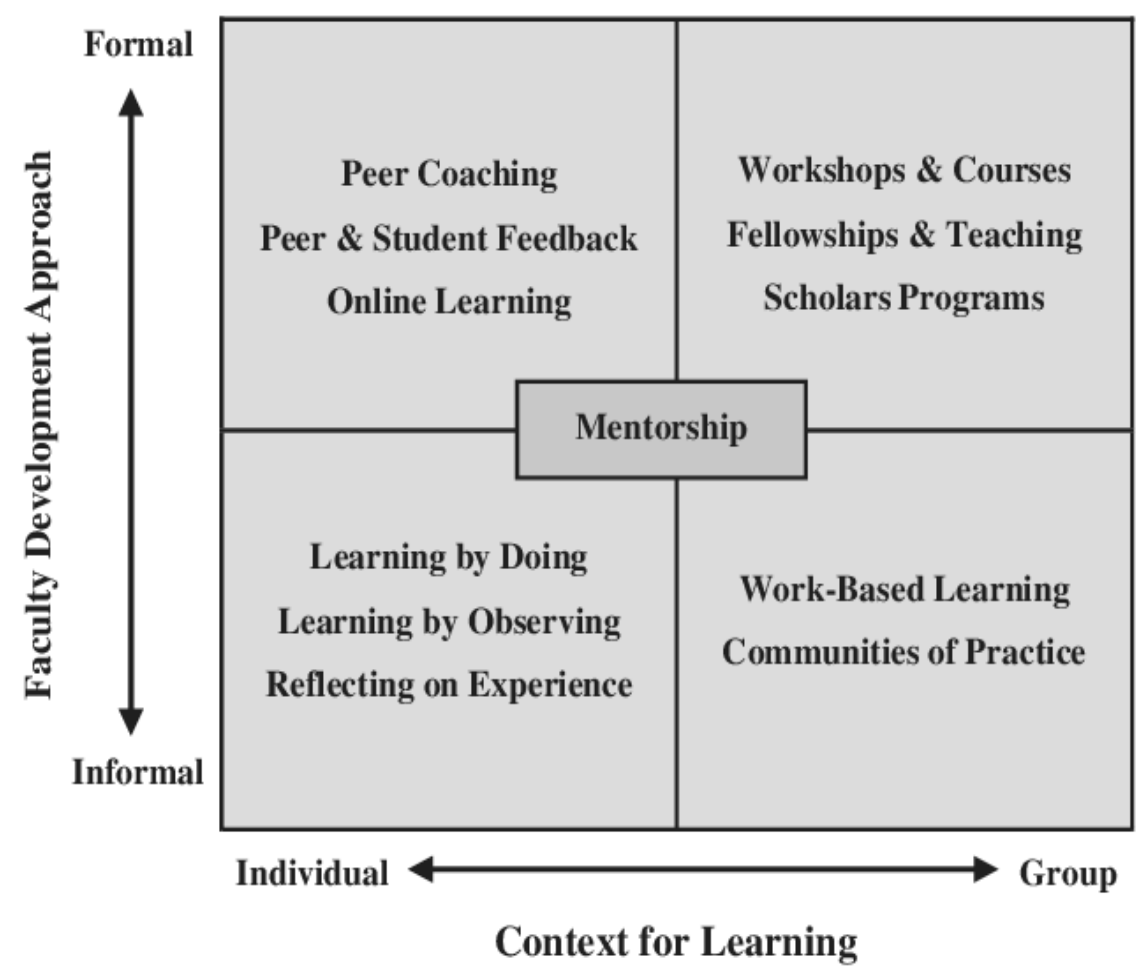

Le Comunità di Pratica si stanno diffondendo nelle Università internazionali come strategia formativa di Faculty Development che valorizza il setting informale, la dimensione gruppale, l'apprendimento nei contesti professionali e la riflessione sulle pratiche come occasione di apprendimento.

Considerato che di solito le CdP si creano spontaneamente e che è abbastanza recente il fenomeno dell'avvio di CdP in modo sistemico, alcuni esperti di Faculty Development, Yvonne Steinert della McGill University di Montreal e Marco Antonio de Carvalho-Filho con René Tio dell'Università di Groningen in Olanda, hanno sentito l'esigenza di definire le 12 raccomandazioni per avviare le comunità di pratica nei contesti del Faculty Development (de CarvalhoFilho, Tio \& Steinert, 2020). 
Questi studiosi tesaurizzano le indicazioni di Wenger e collaboratori (Wenger, et al., 2002) e offrono alcune indicazioni a tutti coloro che desiderano introdurre le CdP nei contesti universitari al fine di riflettere e imparare dalle esperienze didattiche dei docenti stessi (Figura 2).

Fig. 2 - Suggerimenti per realizzare una Comunità di Pratica per il Faculty Development (de Carvalho et al., 2020)

1. Raccogliere un piccolo gruppo di pionieri per lanciare il processo

2. Definire gli scopi e il valore della CdP

3. Partire da un compito o un progetto specifico - rendendolo orientato al problema

4. Mantenere la CdP aperta

5. Invitare intenzionalmente membri con competenze esperte (memoria) e idee nuove (innovazione)

6. Scegliere un facilitatore - primus inter pares

7. Renderla di valore per i membri dell'istituzione

8. Lavorare per assicurarsi il supporto istituzionale

9. Promuoverne la sostenibilità

10. Comunicare i successi

11. Andare online

12. Valutare la CdP

\section{Le Comunità di Pratica per il Faculty Development all'Università di Genova}

L'Università di Genova ha avviato un articolato programma di sviluppo delle competenze didattiche dei propri docenti nel 2016, coordinato dal Gruppo di Lavoro di Ateneo dedicato all'Insegnamento e Apprendimento (GLIA) (Lotti, 2020). Tra le varie iniziative previste vi erano anche le Comunità di Pratica, iniziate nel 2017 in tutte le cinque scuole dell'ateneo genovese: Scuola di Scienze Sociali, Scuola di Scienze Mediche e farmaceutiche, Scuola di Scienze Umanistiche, Scuola Politecnica e Scuola di Scienze Matematiche, Fisiche e Naturali.

Il GLIA ha inteso la Comunità di Pratica come un gruppo di docenti desiderosi di condividere la riflessione sulle strategie formative e valutative utilizzate nel contesto universitario, al fine di migliorare la propria pratica professionale. 
A differenza delle Faculty Learning Communities americane, che si incontrano per affrontare un determinato argomento per la durata di un anno accademico, le CdP genovesi hanno la caratteristica di gruppi di mutuo aiuto permanenti, centrate sui partecipanti, sulle loro esperienze, problematiche e pratiche didattiche. Il ruolo del facilitatore è quello di attivare le energie dei partecipanti al fine di creare, prima di tutto, un gruppo e, successivamente, dare empowerment ai partecipanti.

Il GLIA ha fissato alcuni requisiti per l'avvio delle CdP:

- la numerosità dei partecipanti, che non deve essere superiore a venti;

- la conduzione in capo a una coppia di facilitatori;

- la cadenza mensile;

- la durata di circa un paio di ore;

- la volontarietà di iscrizione e partecipazione.

La coppia di facilitatori, scelti all'interno del GLIA stesso, ha il compito di favorire la presentazione dei partecipanti, la comunicazione delle esperienze $o$ pratiche in modo ordinato, il rispetto dei tempi, la partecipazione di ogni componente del gruppo, la valutazione finale dell'incontro, il rispetto delle posizioni di tutti, il non giudizio, il buon funzionamento del gruppo.

\subsection{Obiettivo della ricerca}

L'Università di Genova ha avviato le CdP nel 2017 nelle cinque scuole, ma, come già anticipato, esse si sono svolte in modo abbastanza disomogeneo per durata, composizione, modalità di conduzione e gestione.

Il GLIA ha sentito la necessità di comprendere meglio e individuare quali sono i fattori che favoriscono il buon funzionamento e la percezione di utilità ed efficacia delle Comunità di Pratica genovesi.

In particolare, il GLIA voleva analizzare in profondità una Comunità di pratica, quella della Scuola di Scienze Matematiche, Fisiche e Naturali, che si caratterizza per regolarità e costanza nel tempo.

\subsection{Materiali e Metodi}

Per raggiungere l'obiettivo dell'indagine, che consiste nel comprendere quali sono i fattori che favoriscono il buon funzionamento delle Comunità di Pratica genovesi, e in particolare la CdP di Scienze Matematiche, Fisiche e Naturali, il GLIA ha deciso di raccogliere le impressioni e i pareri dei membri 
delle Comunità di pratica tramite un questionario, somministrato ai partecipanti e ai facilitatori, che indagasse alcune aree di interesse.

Il questionario, adattato da quello di Olivier Serrat (Serrat, 2017), era articolato in sette sezioni (area di interesse, membri, struttura e processo, flusso di energia, risultati percepiti, risorse e valori) e comprendeva ventinove domande chiuse e sei aperte. Le domande chiuse erano composte da 16 domande con risposta a scelta multipla, la cui risposta consisteva di una scala di Likert a 5 punti $(1=$ non sono completamente d'accordo; $5=$ sono completamente d'accordo), e 13 domande con risposta a scelta multipla a cui era possibile dare una sola risposta o tre risposte in alcuni casi. Il questionario, realizzato su Google Form, è stato inviato tramite posta elettronica ai facilitatori delle comunità di pratica con la richiesta di inoltrarlo agli iscritti alle rispettive Comunità di Pratica a giugno 2020 .

\subsection{Risultati}

I rispondenti sono stati 50 docenti dell'ateneo genovese.

I risultati mostrano che i partecipanti sono per lo più docenti strutturati $(34 \%$ professori associati, 32,6\% ricercatori, 9,3\% professori ordinari) mentre solo un $25 \%$ è composto da professori a contratto, assegnisti o dottorandi.

Quasi la metà dei partecipanti $(47,7 \%)$ ha iniziato a partecipare nell'anno di avvio delle CdP, anno accademico 2017/18, il 13,6\% nell'a.a. 2018/19, e il $38,6 \%$ nell'a.a. $2019 / 20$

Le motivazioni che spingono a partecipare alle CdP sono rintracciabili nel desiderio di rimanere aggiornati sui temi della didattica universitaria $(38,6 \%)$ e raggiungere obiettivi pertinenti alla propria funzione docente $(27,3 \%)$.

Le ragioni del successo delle $\mathrm{CdP}$ risiedono nel trovarvi un ambiente informale, accogliente e socievole $(76,8 \%)$ e la volontà dei suoi componenti di condividere conoscenze, esperienze e problematiche $(90,7 \%)$. Inoltre, i partecipanti apprezzano che vi sia un atteggiamento collaborativo e di condivisione delle conoscenze $(64,3 \%)$, la guida di un facilitatore dedicato e appassionato $(14,3 \%)$, un clima di fiducia nei rapporti umani $(14,3 \%)$. Un altro aspetto importante è che ogni partecipante si sente spinto a condividere ciò che ha maturato durante le esperienze didattiche sul campo $(83,7 \%)$.

Alla domanda su cosa potrebbe fare l'ateneo per aumentare la partecipazione alle $\mathrm{CdP}$, i docenti intervistati chiedono di incoraggiare sistematicamente tutti i docenti a partecipare alla $\mathrm{CdP}(22 \%)$, fornire incentivi ai partecipanti 
(19,5\%), riconoscere il tempo dedicato alle CdP come attività didattica da inserire nei propri registri didattici $(14,6 \%)$.

In sintesi, $i$ docenti che frequentano le $\mathrm{CdP}$ le considerano una esperienza positiva e le definiscono con la metafora di un alveare; una "auberge espagnole" dove ognuno porta ciò che ha e condivide, magari un po' caotica, ma comune; una fabbrica di idee; una Comunità di Curiosi $(\mathrm{CdC})$; un luogo di incontro, condivisione e crescita.

Con riferimento alla Scuola di Scienze Matematiche, Fisiche e Naturali, i partecipanti che hanno risposto al questionario sono stati 18 , di cui il $50 \%$ è professore associato, il 25\% ricercatore a tempo indeterminato, il 19\% ricercatore a tempo determinato di tipo b), e il restante $6 \%$ ricercatore a tempo determinato di tipo a). La grande maggioranza di costoro $(83,3 \%)$ ha iniziato a frequentare la CdP subito al suo avvio nell'a.a. 2017/18, e un altro gruppetto si è inserito nell' anno successivo. Tutti e diciotto i rispondenti dicono di partecipare alla maggioranza delle riunioni che si svolgono ogni mese e durano due ore.

Il 72,2\% dei partecipanti afferma di partecipare alla Comunità di Pratica perché vengono affrontati temi di suo interesse; la grande maggioranza $(88,8 \%)$ afferma di apprezzare la volontà dei componenti di condividere conoscenze, esperienze e problematiche; il $72 \%$ ritiene che nel tempo venga costruito un bagaglio di esperienze e risorse comuni (vedi Figura 3).

Il valore della CdP risiede principalmente nel fatto che diffonde nuove idee per strumenti e metodi di insegnamento/apprendimento/valutazione, per il 50\% dei rispondenti, e che mostra una casistica di buone pratiche, per il 27,8\%.

Il successo della CdP dipende dall'adozione di un atteggiamento collaborativo e di condivisione delle conoscenze $(44,4 \%)$, dall'essere guidati da facilitatori dedicati e appassionati $(27,8 \%)$ e dall'aver instaurato un clima di fiducia e costruito il senso di comunità $(22,2 \%)$.

I fattori motivazionali che spingono i partecipanti a frequentare assiduamente la propria CdP sono essenzialmente tre: la possibilità di restare aggiornati sui temi della didattica universitaria $(38,9 \%)$, l'opportunità di raggiungere obiettivi pertinenti alla propria funzione docente $(33,3 \%)$, e l'apprendimento e lo sviluppo professionale personale $(27,8 \%)$.

Alla domanda su quali fattori siano considerati i più positivi della $\mathrm{CdP}, \mathrm{i}$ partecipanti rispondono compatti: il senso di comunità, lo spirito di condivisione sempre costruttivo e non giudicante, i facilitatori molto competenti, l'incontro di persone che affrontano anche la didattica seriamente e con metodo, l'interdisciplinarietà e il clima informale. 
Fig. 3 - Risultati al questionario da parte della Comunità di Pratica della Scuola di Scienze Matematiche, Fisiche e Naturali dell'Università di Genova (rispondenti n. 18)

\begin{tabular}{|c|c|c|c|c|c|}
\hline $\begin{array}{l}\text { Domanda del questionario. } \\
\text { Partecipo alla comunità di pratica perché: }\end{array}$ & $\begin{array}{c}1 \\
\%\end{array}$ & $\begin{array}{l}2 \\
\%\end{array}$ & $\begin{array}{l}3 \\
\%\end{array}$ & $\begin{array}{l}4 \\
\%\end{array}$ & $\begin{array}{l}5 \\
\%\end{array}$ \\
\hline vengono affrontati temi di mio interesse & 0 & 0 & 0 & 27,2 & 72,2 \\
\hline mi dà un senso di appartenenza & 0 & 11,1 & 16,7 & 27,2 & 44,4 \\
\hline mi permette di costruire relazioni con altri miei colleghi & 0 & 0 & 22,2 & 33,3 & 44,4 \\
\hline il mio lavoro quotidiano trae beneficio dalle relazioni ivi stabilite & 0 & 5,6 & 16,7 & 44,4 & 33,3 \\
\hline $\begin{array}{l}\text { è guidata dalla volontà dei suoi componenti di condividere conoscenze, } \\
\text { esperienze e problematiche }\end{array}$ & 0 & 0 & 5,6 & 5,6 & 88,9 \\
\hline $\begin{array}{l}\text { mi spinge a condividere le conoscenze che ho maturato sul campo della } \\
\text { didattica }\end{array}$ & 0 & 0 & 11,1 & 33,3 & 55,6 \\
\hline nel tempo costruiamo un bagaglio di esperienze e risorse comuni & 0 & 0 & 0 & 27,8 & 72,2 \\
\hline permette di rompere le barriere di comunicazione tra i suoi componenti & 16,7 & 11,1 & 16,7 & 16,7 & 38,9 \\
\hline perché vi è un ambiente informale, accogliente e socievole & 0 & 0 & 11,1 & 27,8 & 61,1 \\
\hline $\begin{array}{l}\text { perché abbiamo anche uno spazio condiviso sulla piattaforma di ateneo } \\
\text { (aulaweb) }\end{array}$ & 27,8 & 16,7 & 38,9 & 11,1 & 5,6 \\
\hline $\begin{array}{l}\text { mi aiuta a raggiungere risultati migliori (qualità, produttività, soddisfazione } \\
\text { degli studenti) }\end{array}$ & 0 & 5,6 & 22,2 & 33,3 & 38,9 \\
\hline $\begin{array}{l}\text { mi aiuta a raggiungere risultati migliori nel mio ambito lavorativo e scienti- } \\
\text { fico disciplinare }\end{array}$ & 16,7 & 16,7 & 22,2 & 33,3 & 11,1 \\
\hline $\begin{array}{l}\text { mi aiuta a raggiungere risultati migliori a livello nazionale nelle mie reti di } \\
\text { pari (associazioni o società scientifiche, ecc.) }\end{array}$ & 33,3 & 38,9 & 16,7 & 11,1 & 0 \\
\hline $\begin{array}{l}\text { permette di far emergere e codificare conoscenze implicite ed esplicite che } \\
\text { poi possono essere sistematizzate e applicate }\end{array}$ & 0 & 11,1 & 11,1 & 38,9 & 38,9 \\
\hline $\begin{array}{l}\text { permette di apprendere e costruire conoscenza partendo dalle esperienze } \\
\text { vissute }\end{array}$ & 0 & 0 & 5,6 & 38,9 & 55,6 \\
\hline rafforza la collaborazione tra i dipartimenti, gli uffici e le unità & 5,6 & 11,1 & 22,2 & 38,9 & 22,2 \\
\hline
\end{tabular}

Anche se la CdP sembra funzionare molto bene, comunque vi sono spazi di miglioramento: i partecipanti auspicano un maggior coinvolgimento di componenti esterni e un riconoscimento formale da parte dell'ateneo.

Per attrarre nuovi membri, i rispondenti suggeriscono di comunicare maggiormente l'esistenza delle Comunità di Pratica ai neoassunti e poi nei consigli di corso di studi e nei consigli di dipartimento. 
Il riconoscimento formale della partecipazione alle Comunità di Pratica rientra in una politica più ampia di valorizzazione dell'attività didattica universitaria rispetto all'attività di ricerca, che permane come unico indicatore di valutazione. A tal fine i partecipanti chiedono che l'ateneo possa supportare maggiormente, riconoscendo il tempo dedicato da coloro che conducono le $\mathrm{CdP}(33 \%)$ e fornendo incentivi e premi per i lavori significativi svolti nelle CdP $(27,8 \%)$.

Alla domanda "Quali altre raccomandazioni hai per rafforzare l'efficacia della $C d P$ " i rispondenti scrivono che a volte sarebbe il caso di organizzare CdP per aree disciplinari omogenee, per poter avere indicazioni settoriali; altri suggeriscono di invitare gli studenti; altri chiedono di poter inviare qualche membro della CdP all'estero in sedi da prendere ad esempio per migliorare la didattica.

\subsection{Discussione dei risultati}

Alla luce dei risultati del questionario inviato ai partecipanti alle Comunità di Pratica dell'ateneo genovese a giugno 2020, emerge che la maggioranza dei partecipanti alle $\mathrm{CdP}$ sono docenti strutturati con contratto a tempo indeterminato, con una maggioranza di professori associati.

Le motivazioni che spingono i docenti a partecipare sono essenzialmente il desiderio di affrontare temi ritenuti di proprio interesse, di costruire relazioni con altri colleghi, di mantenersi aggiornati sui metodi di insegnamento-apprendimento-valutazione nell'ambito della didattica universitaria.

I fattori che sembrano favorire il buon funzionamento di una Comunità di Pratica sono la volontà dei partecipanti di condividere conoscenze, esperienze e pratiche, confermando quanto descritto ampiamente in letteratura (Wenger, et al. 2002) sui tre elementi irrinunciabili di una Comunità di pratica: campo di interesse, comunità e pratica. Anche il ruolo dei facilitatori competenti ha un'importanza decisiva e conferma gli studi di Wenger sulla crucialità del coordinatore di comunità come figura cardine del processo di pianificazione, conduzione del gruppo, comunicazione interna ed esterna tra gruppo e organizzazione di appartenenza.

Rispetto ai fattori che potrebbero rafforzare le Comunità di Pratica emerge con forza il tema del riconoscimento formale da parte dell'ateneo, da intendersi come valorizzazione della didattica universitaria al pari della ricerca scientifica. 


\section{Conclusioni}

Le Faculty Learning Communities sono dunque una forma di Comunità di Pratica presenti nelle Università, dove peraltro si possono trovare anche le Comunità di Pratica. Entrambe sono strategie formative molto interessanti ed efficaci che aiutano a sviluppare un senso di appartenenza all'istituzione, a superare l'isolamento, a rafforzare il senso di auto-efficacia e favoriscono lo sviluppo delle competenze didattiche e valutative dei docenti.

\section{Bibliografia}

Cox, M.D. (2004). Introduction to faculty learning communities. In M.D. Cox, New directions for teaching and learning, n. 97, (pp. 5-23). San Francisco: Wiley periodicals, Inc.

De Carvalho-Filho, M.A., Tio, R.A., \& Steinert, Y. (2020). Twelve tips for implementing a community of practice for faculty development. Medical Teacher, 42(2), 143149.

Engin, M., \& Atkinson, F. (2015). Faculty Learning Communities: a model for supporting curriculum changes in Higher Education. International Journal of Teaching and Learning in Higher Education, 27(2), 164-174.

Kolb, A. (1984). Experiential Learning: experience as the source of Learning and Developing. Englewood Cliffs, NJ: Prentice Hall.

Lotti, A. (2020) L'esperienza del GLIA dell'Università di Genova. In A. Lotti \& P.A. Lampugnani (a cura di). Faculty Development in Italia. Valorizzazione delle competenze didattiche dei docenti universitari. Genova: Genova University Press, pp. 205-217.

Schreurs, M.L., Huveneers, W., \& Dolmans, D. (2016). Communities of teaching practice in the workplace: Evaluation of a faculty development program. Medical Teacher, 38(8), 808-14.

Serrat, O. (2017). Surveying Communities of Practice. In O. Serrat, Knowledge Solutions. Singapore: Springer.

Steinert, Y. (2010). Faculty Development: from workshops to communities of practice. Medical Teacher, 32(5), 425-428.

Steinert, Y. (2014). Learning from experience: from workplace learning to communities of Practice. In Y. Steinert (Ed.). Faculty Development in the health professions. A focus on research and practice. New York: Springer.

Yonge, O.J., \& Davidson, S.J (2017). Promoting scholarship and faculty development through Faculty Learning Communities. Quality Advancement in Nursing Education-Avancées en formation infermière. 3(2), article 5.

Wenger, E. (1998). Communities of Practice, Learning, Meaning and Identity. Cambridge: Cambridge University Press. (trad. it.: Comunità di Pratica. Apprendimento, significato e identità. Milano: Raffaello Cortina Editore, 2006) 
Wenger, E., McDermott R., \& Snyder W.M. (2002) Cultivating Communities of Practice. A guide to managing knowledge. Boston: Harvard Business School Press. (trad.it.: Coltivare le comunità di pratica. Prospettive ed esperienze di gestione della conoscenza. Milano: Guerini Next. 2013). 